\title{
THE INFLUENCE OF THIAMINE DEFICIENCY ON PORPHYRIN SYNTHESIS AND PORPHYRIN PROTEINS
}

\author{
S. HAUSCHILDT ${ }^{1}$ \\ Institut für Ernährungswissenschaft I der Justus-Liebig-Universität Giessen
}

(Received September 8, 1975)

\begin{abstract}
Summary 1. Studies carried out in cell-free rat-liver extracts showed an inhibitory effect of thiamine deficiency on porphyrin synthesis.

2. Extracts of normal and thiamine-deficient rat livers were incubated with the cofactors and substrates of the thiamine pyrophosphate-dependent enzyme, 2-oxoglutarate glyoxylate carboligase. There was a marked inhibition of porphyrin synthesis when the substances were added either individually or together.

3. Thiamine deficiency resulted in a decrease of haemoglobin concentration and catalase activity whereas a rise in tryptophanpyrrolase activity and cytochrome $b_{5}$ and $\mathbf{P}_{450}$ concentration was observed.
\end{abstract}

Thiamine deficiency increases the blood level of glyoxylate as well as of pyruvate (1-3). The oxygen uptake by thiamine-deficient animal tissues metabolising glucose in vitro is also reduced (4). Glyoxylate is highly toxic and reacts with malate to form oxalomalate which inhibits the aconitase [citrate (isocitrate) hydrolyase EC 4.2.1.3] and hence blocks the tricarboxylic acid cycle $(5,6)$. There is also evidence that glyoxylate itself may inhibit some of the other reactions of the tricarboxylic acid cycle (7). Thus, glyoxylate accumulation may be one of the factors which cause death in severe thiamine deficiency.

Glyoxylate is formed from glycine by transamination, by oxidative deamination, by the oxidation of glycollate, and from L-hydroxyproline via 2-oxo-4hydroxyglutarate. It is either oxidized to oxalate, reduced to glycollate, transaminated to glycine or decarboxylated by 2-oxoglutarate: glyoxylate carboligase. The 2-oxoglutarate: glyoxylate carboligase is a thiamine pyrophosphate-dependent enzyme which catalyses the synergistic decarboxylation of 2-oxoglutarate and glyoxylate (8-10). KocH et al. (11) developed a radiochemical assay for the enzyme and identified the reaction product as 2-hydroxy-3-oxoadipate which

${ }^{1}$ Present address: Physiologisch-Chemisches Institut, Lehrstuhl II, Prof. Dr. K. Brand, 8520 Erlangen, Egerlandstraße 7, FRG 
decarboxylates to yield 5-hydroxy-4-oxovalerate. The mechanism of the reaction has also been investigated by SCHLOSSBERG et al. (12), and by KAWASAKI et al. (13). SCHLOSSBERg et al. (14) and SAITO and coworkers (15) concluded that 2-oxoglutarate: glyoxylate carboligase is the same enzyme as oxoglutarate dehydrogenase [2-oxoglutarate: lipoate oxidoreductase (acceptor acylating) EC 1.2.4.2]. The oxidation product of 5-hydroxy-4-oxovaleric acid is 4,5-dioxovaleric acid which has been shown to be partially converted to 5 -amino-4-hydroxyvaleric acid ( $\delta$ amino laevulinic acid) by KISSEL and HeILMEYER (16). Thus, a failure to metabolise glyoxylate via this pathway might reduce $\delta$-amino laevulinic acid production and hence the formation of porphyrins.

Abnormal glyoxylate accumulation which inhibits the tricarboxylic acid cycle (5) could also reduce the supply of succinate from the tricarboxylic acid

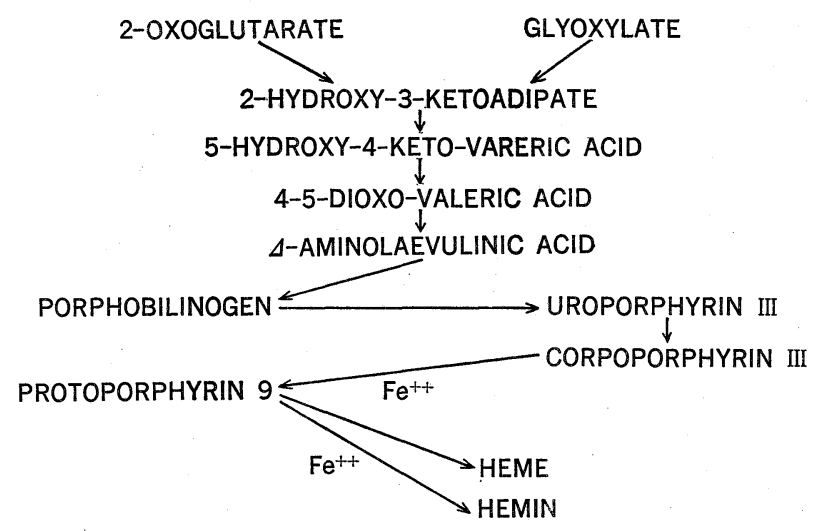

Fig. 1. Possible pathway of glyoxylate.

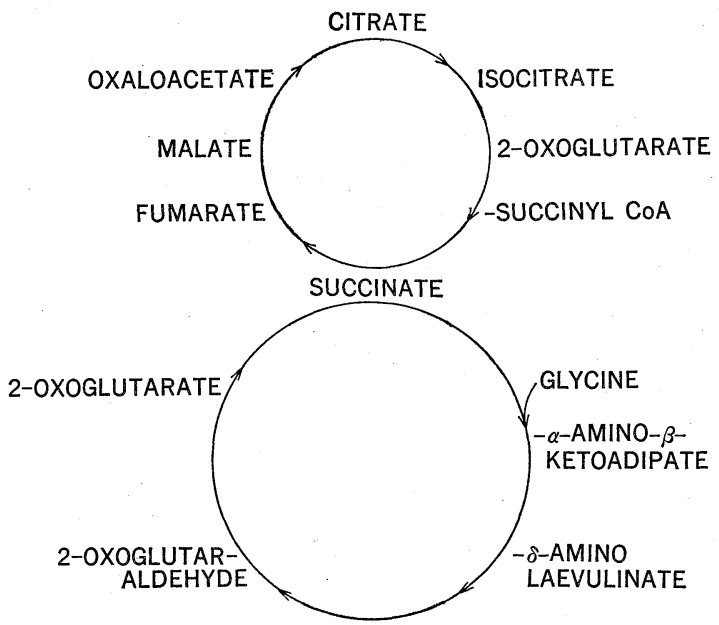

Fig. 2. Linkage of the succinate glycine and tricarboxylic acid cycles through succinate. 
cycle to the succinate glycine cycle (17) for porphyrin synthesis. These metabolic inter-relationships are summarised in Figs. 1 and 2.

The present paper concerns a study designed to investigate the effect of thiamine deficiency on the production of porphyrins based on:

1) the rate of porphyrin synthesis by liver tissue in vitro;

2) the activity of the porphyrin containing enzymes catalase $\left(\mathrm{H}_{2} \mathrm{O}_{2}: \mathrm{H}_{2} \mathrm{O}_{2}\right.$ oxidoreductase EC 1.11.1.6) and tryptophan pyrrolase (tryptophan oxygenase EC 1.99.2.c);

3) the production of haemoglobin and cytochromes $b_{5}$ and $P_{450}$.

\section{MATERIALS AND METHODS}

Thiamine deficiency was induced in female albino rats (Wistar) that had been fed a conventional diet devoid of thiamine (18) weighing log; they were taken for study as soon as they exhibited convulsions. Pair fed animals received the same amount of diet as the thiamine-deficient animals but with added thiamine. Fasted animals received $5 \mathrm{~g}$ of control diet for 7 days, and glyoxylate-treated animals were given a sodium glyoxylate solution $(1 \% \mathrm{w} / \mathrm{v})$ instead of drinking water. The porphyrin synthesizing ability of cell-free rat liver extracts was determined by the method of YAMASAKI and MORIYAMA (19). The animals were decapitated, the livers quickly removed with a prefrozen Wollenberg clamp and put into liquid nitrogen. The livers were ground thoroughly in a pre-cooled iron mortar. The powder was quickly weighed and transferred to a ceramic mortar containing frozen perchloric acid snow $(9 \% \mathrm{w} / \mathrm{v})$. The perchloric acid snow and powdered liver were mixed thoroughly, and the mixture was allowed to thaw in ice for 2 hr. After the mixture had thawed completely, it was transferred to a centrifuge tube, centrifuged and the supernatant solution neutralised with $5 \mathrm{M} \mathrm{K}_{2} \mathrm{CO}_{3}$. After keeping the supernatant in ice for $1 \mathrm{hr}$, the $\mathrm{KCIO}_{4}$ was separated by centrifugation and the supernatant was taken for analysis. The tryptophan pyrrolase activity of the cell free liver extracts was estimated by measuring the amount of kynurenine formed during aerobic incubation of rat liver homogenate with Ltryptophan (20). Haemoglobin concentration and catalase activity were determined using capillary blood by the method of Van KAMPEN and ZIJLSTRA (21) and BERGMEYER (22), respectively.

For the assay of cytochrome $b_{5}$ and $P_{450}$, modified methods of KLINGENBERG (23) and OMURo and SAITo (24) were used, respectively.

\section{RESULTS}

Porphyrin synthesis was reduced in the thiamine-deficient and in the pair-fed controls, and most markedly in the animals given glyoxylate (Table 1). The possible relationship of this phenomenon to 2-oxoglutarate: glyoxylate carboligase activity was investigated by measuring porphyrin synthesizing ability in the 
Table 1. Porphyrin synthesis in rat liver extracts (All values-arbitrary units of porphyrin synthesized-are expressed per mg protein).

\begin{tabular}{rlcc}
\hline \multicolumn{1}{c}{ Animal } & Number used & Mean \pm S.D. \\
\hline I. Control rats & 18 & $13.9 \pm 4.8$ \\
II. Thiamine-deficient rats & 33 & $10.5 \pm 4.1$ \\
III. Pair-fed rats & 8 & $10.0 \pm 1.6$ \\
IV. Glyoxylate-treated rats & 7 & $6.3 \pm 1.4$ \\
\hline
\end{tabular}

Values represent the means of duplicate determinations on the number of animals tested. Significance determined by t-test on the differences between the groups I, II, III, IV.

I-II: $P<0.01$. I-IV: $P<0.001$.

Table 2. Inhibition of porphyrin synthesis in rat liver extracts by addition of the substrates and cofactors for carboligase. (inhibition = per cent of control)

\begin{tabular}{|c|c|c|c|}
\hline & Compound & $\begin{array}{c}\text { I } \\
\text { Control rats }\end{array}$ & Thiamine-deficient rats \\
\hline a) & $\begin{array}{l}\text { Glyoxylate } \\
\left(6.7 \times 10^{-4} \mathrm{M}\right)\end{array}$ & $55 \pm 11$ & $60 \pm 11 \quad(7)$ \\
\hline b) & $\begin{array}{l}\text { 2-Oxoglutarate } \\
\left(6.7 \times 10^{-4} \mathrm{M}\right)\end{array}$ & $43 \pm 6(8)$ & $69 \pm 15$ \\
\hline c) & $\begin{array}{l}\text { TPP } \\
(360 \mu \mathrm{M})\end{array}$ & $43 \pm 16(12)$ & $25 \pm 15$ \\
\hline d) & $\begin{array}{l}\mathrm{Mg} \mathrm{SO}_{4} \\
\left(3.3 \times 10^{-3} \mathrm{M}\right)\end{array}$ & $20 \pm 20(11)$ & $-1.4 \pm 11(10)$ \\
\hline \multicolumn{2}{|c|}{ e) + b) + c) + d) } & $43 \pm 10 \quad(6)$ & $67 \pm 10 \quad(6)$ \\
\hline
\end{tabular}

Values represent the means ( \pm standard derivations) of duplicate determinations on the number of animals shown in parenthesis. Significance determined by t-test on the differences between the groups I and II.

b) I-II: $P<0.001$

c) I-II: $P<0.02$

d) I-II: $P<0.01$. a) +b) + c) + d), I-II: $P<0.002$.

Table 3. Hemoglobin, hematocrit and erythrocytes in the capillary blood of control, thiamine-deficient and glyoxylate-treated rats.

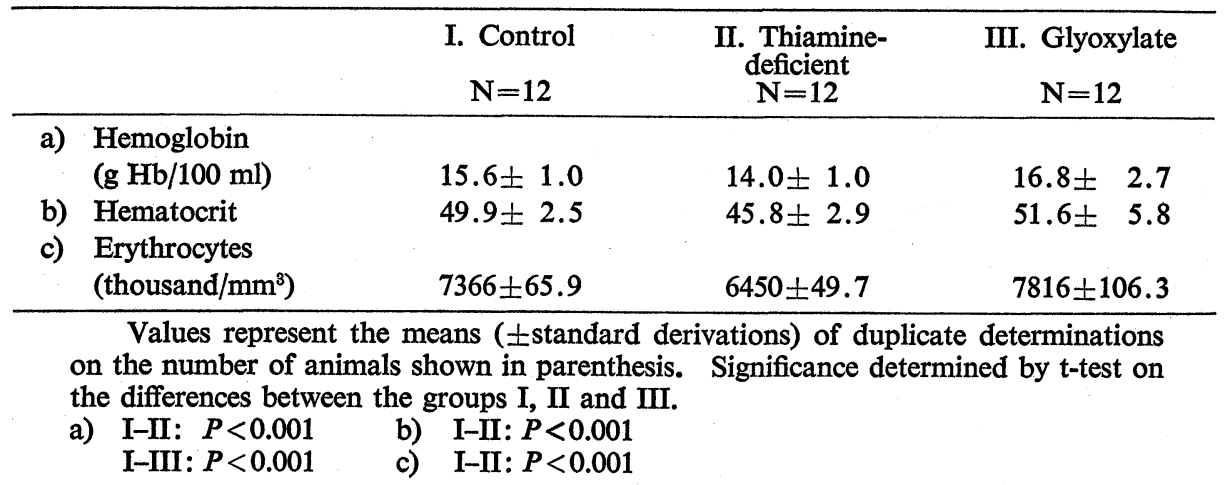


presence of the substrates and co-factors for the carboligase reaction. Adding the substrates and co-factors either separately or together reduced porphyrin synthesis, and the effects were more marked in the thiamine-deficient animals (Table 2).

Thiamine deficiency was associated with a reduced haemoglobin concentration, haematocrit and erythrocyte count. Glyoxylate feeding did not affect these

Table 4. Catalase activity in capillary blood of control, thiamine-deficient, pair-fed and glyoxylate-treated rats (All values are expressed in units per gram hemoglobin).

\begin{tabular}{rlc}
\hline \multicolumn{1}{c}{ Animal } & Mean \pm standard deviation \\
\hline I & Control rats & $3446 \pm 614(18)$ \\
II & Thiamine-deficient rats & $2354 \pm 431(28)$ \\
III & Pair-fed rats & $2689 \pm 461(10)$ \\
IV & Glyoxylate-treated rats & $2540 \pm 398(9)$ \\
\hline
\end{tabular}

Values represent the means of duplicate determinations on the number of animals shown in parenthesis. Significance determined by $t$-test on the difference between the groups I, II, III and IV.

I-II: $P<0.001 \quad$ I-IV: $P<0.001$

I-III: $P<0.001 \quad$ II-IV: $P<0.01$

Table 5. Tryptophan pyrrolase activity in liver homogenate of control, thiamine-deficient, pair-fed and glyoxylate-treated rats (All values are expressed in $\mu_{\mathrm{M}}$ kynurenine per gram dry weight per hour).

\begin{tabular}{rlc}
\hline \multicolumn{1}{c}{ Animal } & Mean \pm standard deviation \\
\hline I & Control rats & $1.1 \pm 0.5(24)$ \\
II & Thiamine-deficient rats & $7.8 \pm 2.6(26)$ \\
III & Pair-fed rats & $4.0 \pm 2.0(12)$ \\
IV & Glyoxylate-treated rats & $4.5 \pm 2.7(13)$ \\
\hline
\end{tabular}

Values represent the means of duplicate determinations on the number of animals shown in parenthesis. Significance determined by t-test on the differences between the groups I, II, III and IV.

I-II : $P<0.001 \quad$ II-III: $P<0.001$

I-III: $P<0.001 \quad$ II-IV: $P<0.001$

I-IV: $P<0.001$

Table 6. Content of cytochromes $b_{5}$ and $\mathbf{P}_{450}$ in rat liver microsomes (All values are expressed in $\mathrm{m} \mu$ moles per $\mathrm{mg}$ protein).

\begin{tabular}{|c|c|c|c|}
\hline & & a) $P_{450}$ & b) $b_{5}$ \\
\hline I. & $\begin{array}{l}\text { Control rats } \\
(n=17)\end{array}$ & $0.39 \pm 0.02$ & $0.36 \pm 0.02$ \\
\hline II. & $\begin{array}{l}\text { Thiamine-deficient rats } \\
(n=21)\end{array}$ & $0.75 \pm 0.11$ & $0.62 \pm 0.08$ \\
\hline
\end{tabular}


parameters (Table 3). The catalase activity of capillary blood was also reduced in the thiamine-deficient, the pair-fed and in the glyoxylate-treated animal (Table 4), whereas tryptophan pyrrolase activity was increased in all three groups of experimental animals (Table 5). The tissues of thiamine-deficient animals also contained more cytochromes than did tissues from the control animals (Table 6).

\section{DISCUSSION}

Severe thiamine deficiency produces a complicated series of biochemical derangements, the relative importance of which varies at different stages in the evolution of the vitamin-deficient state. Thiamine deficiency reduces food intake, and it may be difficult to disentangle the specific effects of the vitamin deficiency from those of malnutrition even with the help of careful pair feeding techniques. Glyoxylate accumulates in severe thiamine deficiency $(1-4,18,25)$ and has widespread toxic effects which probably arise, at least in part, from its ability to undergo non-enzymic condensation reactions with, for example, sulfhydryl compounds (26) and malate (6).

The present observation that the reduction in porphyrin synthesis observed in the thiamine-deficient animals was increased in the controls given glyoxylate (Table 1) suggests that the effect was due to glyoxylate accumulation. This could arise from tricarboxylic acid cycle inhibition with reduced availability of succinyl-CoA condensation with glycine to form $\alpha$-amino- $\beta$-ketoadipic acid and hence $\delta$-amino laevulinic acid.

YAMASAKI and MORIYAMA (19) found that incubating cell-free bacterial extracts with the substrates and co-factors for the glyoxylate: 2-oxoglutarate carboligase reaction inhibited porphyrin synthesis in the extract. Similar results were obtained in the present work, and it is of interest that restoring the carboligase activity of the thiamine-deficient tissue did not restore porphyrin synthesis to normal levels but inhibited it further. The product of the carboligase reaction, 2-hydroxy-3-oxoadipate is oxidised to 5-hydroxy-4-oxovalerate and hence to 4,5-dioxovalerate which KisSel and HeILMEYer (16) found to be a precursor of 5-amino-4-oxovalerate ( $\delta$-amino-laevulinate). Thus, increased porphyrin synthesis from this substrate might have been expected in the presence of increased glyoxylate: 2-oxoglutarate carboligase activity. However, the studies with microorganisms (19) suggest that 5-hydroxy-4-oxovaleric acid inhibits porphobilinogen synthase [5-aminolaevulinate hydrolyase (adding 5-aminolaevulinate and cyclizing EC 4.2.1.24] and therefore reduces porphyrin synthesis. Laevulinic (4-oxovaleric) acid also inhibits porphobilinogen synthetase. The present observations suggest that glyoxylate: 2-oxoglutarate carboligase activity may be a factor regulating the rate of porphyrin biosynthesis in mammalian, as well as in bacterial systems, increasing the supply of $\delta$-amino laevulinate independently of the activity of $\delta$-aminolaevulinate synthase but also inhibiting the condensation of two molecules of $\delta$-aminolaevulinate to form porphobilinogen. 
The reduced porphyrin synthesizing ability which was demonstrated in the thiamine-deficient animal's liver tissue was not reflected in the haemoglobin levels and catalase activity. The haemoglobin, erythrocyte counts, and haematocrit levels were all reduced in the thiamine-deficient animals, so that the amount of haemoglobin per red cell was unaltered. These results together with the reduced catalase activity were not altered by glyoxylate feeding, and cannot be specifically ascribed to thiamine depletion.

The increased tryptophan pyrrolase activity and increased cytochrome content of the thiamine-deficient animals' livers may reflect a relatively slow turnover time for these tissue haemoproteins. The complex biochemical effects of thiamine deficiency, and possibly of the secondarily reduced food intake which this produces, may cause death before there is time for the effect of reduced porphyrin synthesizing ability to affect these parameters. As it is known that tryptophan pyrrolase can be induced by tryptophan itself (27) this effect might have been due to a tissue breakdown. The tryptophan pyrrolase activities and cytochrome levels are related to the tissue dry weight and protein content, respectively, so that the possibility that these results reflect loss of other tissue constituents cannot be excluded.

\section{REFERENCES}

1) LiANG, C. C., Biochem. J., 82, 429 (1962).

2) LIANG, C. C., Biochem. J., 83, 101 (1962).

3) Buckle, R. M., Clin. Sci., 25, 207 (1963).

4) LiANG, C. C., Biochem. J., 85, 38 (1962).

5) D’Abramo, F., Romano, M., and Ruffo, A., Biochem. J., 68, 270 (1958).

6) Ruffo, A., Testa, E., Adinolfi, A., and PelizzA, G., Biochem. J., 85, 588 (1962).

7) Ruffo, A., Adinolfi, A., Budillon, G., and CApobianco, G., Biochem. J., 85, 593 (1962).

8) Crawhall, J. C. and Watts, R. W. E., Biochem. J., 85, 163 (1962).

9) Косн, J. and Stokstad, E. L. R., Biochem. Biophys. Res. Comm., 23, 585 (1966).

10) Stewart, P. R. and QuaYle, J. R., Biochem. J., 102, 885 (1967).

11) Koch, J., Stokstad, E. L. R., Williams, H. E., and Smith, L. H., Proc. Nat. Acad. Sci., 57, 1123 (1967).

12) Schlossberg, M. A., Richert, D. A., Bloom, R. J., and Westrfeld, W. W., Biochemistry, 7, 333 (1968).

13) Kawasaki, H., Okuyama, M., and Kikuchi, G., J. Biochem. (Tokyo), 59, 419 (1966).

14) Schlossberg, M. A., Bloom, R. J., Richert, D. A., and Westerfeld, W. W., Biochemistry, 9, 1148 (1970).

15) Saito, T., Tuboi, S., Nishimura, Y., and Kixuchi, G., J. Biochem., 69, 265 (1971).

16) Kissel, H. J. and HeILMeYer, L., Biochim. Biophys. Acta, 177, 78 (1969).

17) Shemin, D., in W. D. McElroy, B. Glass (Editors), A symposium on amino acid metabolism, John Hopkins Press, Baltimore (1955).

18) Hauschildt, S. and Feldheim, W., Internat. Z. Vit.-Ern.-Forschung, 41, 408 (1971).

19) Yamasaki, H. and Moriyama, T., Biochem. Biophys. Res. Comm., 38, 638 (1970).

20) Knox, W. E. and Auerbach, V. H., J. Biol. Chem., 214, 307 (1955).

21) Van Kampen, E. J. and Zislstra, W. G., Clin. Chim. Acta, 6, 538 (1961). 
22) Bergmeyer, H. U., Methods of enzymatic analysis, Verlag Chemie, GmbH. Weinheim/ Bergstr, p. 886 (1963).

23) Kirngenberg, M., Arch. Biochem. Biophys., 75, 376 (1958).

24) Omuro, T. and Sarto, R., J. Biol. Chem., 239, 2370 (1964).

25) LiANG, C. C., Nature (London), 188, 660 (1960).

26) Rao, N. A. N. and Ramakrishnan, T., Biochim. Biophys. Acta, 58, 262 (1962).

27) Knox, W. E., Auerbach, V. H. and Lin, E. C. C., Physiol. Rev., 36, 164 (1956). 